\title{
Categories of transdiagnostic processes: Qualitative data from psychotherapy practitioners
}

\author{
António Farinha-Fernandes ${ }^{1}$ (D) | Nuno Conceição ${ }^{1,2}$ (D) | Rita Silva ${ }^{1}$ \\ ${ }^{1}$ Research Center for Psychological Science (CICPSI), Faculdade de Psicologia, Universidade de Lisboa, Lisboa, Portugal \\ ${ }^{2}$ Applied Psychology Research Center Capabilities \& Inclusion (APPsyCI), ISPA - Instituto Universitário, Lisboa, Portugal
}

Correspondence: António Farinha-Fernandes, Faculdade de Psicologia, Universidade de Lisboa, Alameda da Universidade, $1649-013$ Lisboa, Portugal. Email: antonio.fernandes@campus.ul.pt

\section{1 | INTRODUCTION}

The processes involved in the development, persistence and maintenance of psychopathology have been subject to frequent debate. A particular focus of discussion is the one comparing categorical and dimensional approaches to diverse psychopathological patterns (e.g. Hopwood et al., 2018; Kraemer, Noda, \& O'Hara, 2004; Widiger, 1992). Categorical systems rely on signals (observed by the clinician) and symptoms (reported by the patient), which are viewed as important indicators of the presence of a disorder (such as in the DSM-V or the ICD-10). There are specific diagnostic criteria for each disorder, and a patient is diagnosed with one or more disorders when meeting those criteria. Dimensional systems, on the other hand, rate patients on different dimensions that aim to consider important individual variability and avoid a dichotomous decision between a present and an absent diagnosis. An individual is thus assessed in a set of domains, which arguably allows to draw a deeper profile of impairment or severity across them (Helzer, Kraemer, \& Krueger, 2006; Rodriguez-Seijas, Eaton, \& Krueger, 2015).

Traditional categorical systems have been subject to extensive criticism in recent years (Hengartner \& Lehmann, 2017; Hopwood et al., 2018), in favour of dimensional approaches (e.g. the RDoC initiative in Yee, Javitt, \& Miller, 2015). These dimensional approaches, however, can be seen as complementary, instead of substitutive. Psychopathology is widely acknowledged as a dynamic construct, which has been leading to the discussion of perspectives that can complement the categorical view, thus allowing for a better understanding of its underlying mechanisms (Nelson, McGorry, Wichers, Wigman, \& Hartmann, 2017). While some authors consider that categorical or dimensional approaches are more appropriate depending on the clinical circumstances and research questions being addressed
(Kraemer, Noda, \& O'Hara, 2004), others argue that a dimensional component should be added to the traditional categorical systems, thus preserving their existence (Helzer, Kraemer, \& Krueger, 2006).

One such dimensional perspective, for which discussion has been reignited in the literature, is the transdiagnostic perspective. This perspective can be seen as an alternative to the widespread division of psychological disorders in categorical and discrete entities, as well as to diagnosis-based interventions. As such, it can offer new insights when approaching the underlying processes implicated in mental health (Dalgleish, Black, Johnston, \& Bevan, 2020). A particular transdiagnostic approach consists of studying and conceptualising common processes underlying the development or maintenance of different disorders (for an example, see the shared mechanisms approaches as discussed in Sauer-Zavala et al., 2017). It is suggested that such a transdiagnostic scope could help to reduce the complexity caused by the high levels of comorbidity observed between various categorical diagnoses (Rodriguez-Seijas et al., 2015; Taylor \& Clark, 2009).

Although this transdiagnostic perspective has recently been gaining more attention, some authors point to its historical roots (Mansell, Harvey, Watkins, \& Shafran, 2009; Nolen-Hoeksema \& Watkins, 2011). For instance, both the psychoanalytic view and the first behavioural approaches discussed general principles (e.g. defence mechanisms in the former, operant and classical conditioning in the latter) that could be applied to several clinical patterns, such as phobia, depression or schizophrenia (reviewed in Nolen-Hoeksema $\&$ Watkins, 2011). This idea has been examined again in more recent articles. Serving as an example, Gellatly and Beck (2016) conducted a comprehensive literature review on the role of catastrophic beliefs (originally discussed by Ellis, 1962) as a predictor of several disorders, such as panic, phobia, health anxiety, obsessive-compulsive 
disorder, post-traumatic stress disorder, pain and traumatic brain injury.

Overall, there is a growing agreement on the fact that different disorders involve dysfunctional versions of processes that vary along a continuum in the general population, which are observed across several categories. The transdiagnostic approach thus focuses on such dysfunctional processes, aiming to contribute to the understanding of psychopathology in a more parsimonious way (Harvey et al., 2004; Nolen-Hoeksema \& Watkins, 2011; Rodriguez-Seijas et al., 2015).

In general, transdiagnostic assessment can arguably be simpler and more efficient than traditional assessment, since it allows us to weigh and combine signals and/or symptoms to understand the severity of the problem, to accommodate the heterogeneity of diagnoses and to provide information about transdiagnostic factors (Rodriguez-Seijas et al., 2015). Furthermore, this approach can also help to reach interventions that focus on such transdiagnostic aspects, thus influencing the various disorders that can be associated with them. That is, the identification of transdiagnostic processes can translate directly into potential focuses of intervention. It is assumed that an intervention that reverts the underlying processes of a particular disorder could lead to positive effects on comorbid secondary disorders (Harvey et al., 2004; McEvoy, Nathan, \& Norton, 2009). For example, a study from Feldman, Tung, and Lee (2017) suggests that individual differences in social skills (i.e. cooperation, assertion, responsibility, self-control) may play a role in the association between depressive symptoms and ADHD in youngsters, thus constituting a potential focus of intervention. La Greca, Ehrenreich-May, Mufson, and Chan (2016) also suggest that the prevention of social anxiety and depression in adolescents could benefit from a transdiagnostic intervention, and discuss the importance of considering peer victimisation as a peer risk factor for these disorders. Consequently, the authors developed a transdiagnostic intervention comprising strategies to cope with depression, social anxiety and peer victimisation.

There has been a growing search for models that are able to explain how and why transdiagnostic processes result in the development of different psychopathological patterns, as well as for specific processes that, albeit not necessarily inserted in a model, are potentially transdiagnostic. For example, Harvey et al. (2004) thoroughly reviewed empirical studies about the cognitive and behavioural processes involved in Axis I disorders on the DSM-IV, with the purpose of identifying similar transdiagnostic processes. These authors discuss five general categories of processes: at tentional processes, memory processes, reasoning processes, thought processes and behavioural processes. Baer (2007) later described the practice of mindfulness as a transdiagnostic intervention-related element. Carey (2008) describes the internal perceptual conflict as a transdiagnostic process and discusses how the perceptual control theory can help to understand and resolve this conflict. McEvoy et al. (2009) reviewed 10 treatment trials and a meta-analysis of transdiagnostic interventions, identifying intervention-related transdiagnostic elements, such as psychoeducation, cognitive restructuring, exposure or behavioural experiments. On a different note, Nolen-Hoeksema and Watkins (2011) developed a heuristic for developing transdiagnostic models of psychopathology, explaining the dynamics between risk factors (distal or proximal) and other moderating factors leading to the sequential development of psychopathology. These authors also describe multifinality as the phenomenon by which risk factors lead to multiple comorbid disorders, and divergent trajectories as the phenomenon through which psychopathology expresses itself in different ways for different individuals.

McKay, Fanning, and Ona (2011) also identified seven maladaptive coping strategies that appear to be common to every emotional disorder (e.g. experiential avoidance, rumination or hostility/aggression), as well as eight transdiagnostic intervention elements associated with specific skills (e.g. emotional exposure, mindfulness or interpersonal effectiveness training). Likewise, Barlow and collaborators (2011) created a unified protocol for a cognitive-behavioural transdiagnostic intervention in emotional disorders. The protocol is based on common principles such as restructuring maladaptive cognitive appraisals, changing maladaptive action tendencies, preventing emotional avoidance and using emotional exposure procedures. This model thus addresses the adaptive and functional nature of emotions, aiming to identify and modify maladaptive attempts at emotional regulation, and facilitating the reduction of excessive emotional responses to internal and external cues. Finally, Frank and Davidson (2014) developed a transdiagnostic model for case conceptualisation describing (1) transdiagnostic mechanisms (vulnerability mechanisms such as neurophysiological predispositions or learned responses, and response mechanisms such as experiential avoidance or attributional biases); (2) a method to link these mechanisms with patients' problems; and (3) a step-by-step process to guide therapists through the therapeutic process.

In sum, the previously presented studies and models have contributed to the listing of transdiagnostic processes in two ways: by defining innovative common processes (or known processes that were not previously presented as such), and by reinforcing and supporting the transdiagnostic character of certain processes (when they are acknowledged by various authors).

In spite of the fact that there are several authors discussing the advantages of transdiagnostic approaches, it is important to make sure that these approaches effectively help patients to consider their difficulties and to move towards intervention goals (Dudley, Kuyken, \& Padesky, 2011). In other words, the transdiagnostic perspective might risk compromising its complexity reduction goal if one cannot find a direct link between theory and practice. It is also possible that this view can be considered more disorganised than specific views and categories for each disorder. In fact, the criteria through which a process or intervention can be defined as transdiagnostic are still not clear. Recent articles also reflect an unclear use of different terms when referring to the transdiagnostic elements, such as processes, mechanisms and factors (e.g. Gallagher, 2017; LaRowe, Zvolensky, \& Ditre, 2019; Zelkowitz \& Cole, 2019). Here, we choose to use the transdiagnostic process designation to describe the common aspects across disorders that might contribute to their development and/or maintenance.

Furthermore, given the diversity of clinical patterns and therapeutic approaches, the list of transdiagnostic processes directly 
related to the clinical reality should be far from closed. Other than just immersing oneself in the literature of this specialised area of knowledge, one can also directly address practitioners in a somewhat naturalistic setting. Therefore, we sought clinicians of any orientation in order to explore whether there exists qualitative evidence that supports processes that have been explicitly referred to as transdiagnostic in the literature, as well as other processes that might still be missing such a status. Here, we present an exploratory qualitative study, based on the work conducted by clinicians in their last week of sessions, as a potential contribution to the simplification and applicability of the transdiagnostic perspective.

\subsection{Study goals and research question}

Using a qualitative methodology, we aimed at providing evidence for the recognition of specific transdiagnostic processes by practitioners. We used an exploratory and descriptive approach, relying on reports from the participants to describe and conceptualise the phenomena based on the obtained data.

The main research question was: 'What are the processes acknowledged as transdiagnostic by clinicians?' Aiming to obtain naturalistic information coming from clinicians' daily practice, however, the questions asked to participants were more case-centred, as described in Methods section.

\section{2 | METHODS}

\section{1 | Participants}

To participate in our study, subjects were required to be psychotherapy practitioners, independently of their theoretical orientation and of total years of professional practice. We used a non-probabilistic sampling methodology, from which a convenience sample emerged. Subjects were invited to participate via an email invitation that included a direct link to a questionnaire (further described in the Procedure subsection), which was destined to members of the Society for the Exploration of Psychotherapy Integration (SEPI) and of the Society for Psychotherapy Research (SPR), which were both international societies.

A total of 51 subjects voluntarily participated in the study $\left(M_{\text {age }}=56.16, S D_{\text {age }}=13.68,24\right.$ females). Table 1 shows a general characterisation of our sample, in what pertains to socio-demographic information, level of education and years of professional experience.

\section{2 | Procedure}

Participants were asked to fill in an online questionnaire developed using the Qualtrics platform. First, subjects were presented an informed consent form, which they were asked to read and accept
TABLE 1 Characterisation of the sample

\begin{tabular}{|c|c|c|c|c|}
\hline Variable & $M$ & $S D$ & $n$ & $\%$ \\
\hline Age & $56.16(27-79)$ & 13.68 & & \\
\hline \multicolumn{5}{|l|}{ Gender } \\
\hline Male & & & 27 & 52.94 \\
\hline Female & & & 24 & 47.06 \\
\hline \multicolumn{5}{|l|}{ Nationality } \\
\hline USA & & & 30 & 58.82 \\
\hline Other & & & 21 & 41.18 \\
\hline \multicolumn{5}{|l|}{ Experience } \\
\hline$>25$ years & & & 29 & 56.86 \\
\hline \multicolumn{5}{|l|}{$\leq 25$ years } \\
\hline $15-25$ years & & & 6 & 11.76 \\
\hline $7-15$ years & & & 6 & 11.76 \\
\hline $3.5-7$ years & & & 5 & 9.8 \\
\hline $1.5-3.5$ years & & & 4 & 7.84 \\
\hline$<1.5$ years & & & 1 & 1.96 \\
\hline \multicolumn{5}{|l|}{ Education } \\
\hline $\begin{array}{l}\mathrm{PhD} / \mathrm{PsD} / \\
\mathrm{EdD}\end{array}$ & & & 42 & 82.35 \\
\hline M.S./M.A. & & & 5 & 9.8 \\
\hline M.D. & & & 3 & 5.88 \\
\hline B.S./B.A. & & & 1 & 1.96 \\
\hline
\end{tabular}

prior to participation. An email contact from the researchers was provided at this stage, for clarification of any aspect pertaining to the study. Then, participants were invited to provide the sociodemographic data that allowed us to characterise our sample. After this, they were presented with two qualitative questions, which invited them to evoke one or more patients with whom they had worked in the past week.

The first question aimed at exploring common processes between two or more comorbid disorders/issues in a determined patient of their choosing from their caseload: 'Pick one client/patient from your last week who has at least two different comorbid disorders/issues, but whose problems, or their transformation, reflect a common underlying process'. The second question aimed at exploring common processes between two or more patients with different disorders/issues: 'Pick two or more clients/patients from your last week who have at least two different disorders/issues, but whose problems, or their transformation, reflect a common underlying process'.

Note that, rather than asking clinicians directly what processes they think are transdiagnostic, we deliberately chose to ask them to describe what processes are common to either a single patient with multiple disorders or multiple patients with different disorders. Participants could choose to answer both questions or only one of them, depending on their will and availability to share more or less. We chose to use open-ended questions, since this format allows participants to explore and describe their subjective experience (Hill, Chui, \& Baumann, 2013). This could be formulated as 
a simple, indirect yet focused, clinically anchored task to elicit raw, naturalistic data to be later analysed by us, without demanding from participants any explicit reflection about processes they would think are transdiagnostic. We wanted the data analysis to be conducted on the clinical material they provided, not on their perspectives of what they would themselves consider transdiagnostic.

Participating in this study required approximately 30 minutes, varying in function of the written communication style of each participant, as well as of the quantity of information that they were willing to share. Ethics approval was granted by the faculty's Ethics Committee via written application. Although there were no incentives for participation, a list of transdiagnostic factors, as reviewed by the authors, was displayed for participants to download after completion if they wished to, as an appreciation for their time.

\section{3 | Data analysis}

The collected data were analysed in a qualitative manner, using the thematic analysis methodology (see Braun \& Clark, 2006). This approach allows us to identify, analyse and report relevant patterns or themes in a group of data, such as participants' answers to our open-ended questions. The analysis occurs in six stages: (1) familiarising with the data; (2) creating the first units of meaning based on data that is relevant to the research question; (3) grouping these units of meaning into themes or superior categories; (4) revising the categories that were created and the references that were codified (5) defining and naming the themes that configure a thematic map of the data; and (6) reporting the story told by the data.

The analysis was performed at the semantic level, through the identification of themes and categories that captured participants' descriptive details in relation to transdiagnostic processes. As suggested by Braun \& Clarke (2006), themes were transformed into categories after they had been reviewed at least three times. Furthermore, all categories were formed considering the relevance of the themes for research, even if they were mentioned only once. It is important to mention that the creation of units of meaning was essentially based on the collected information, although some of the superior categories are common to previous research and pre-existing theoretical concepts.

Note that we chose here not to specify which information about which question is being answered because the set of questions consisted of a bundle task or a global means to elicit potentially relevant clinical material. Therefore, by itself, each question alone does not merit to be analysed separately. This task was our way of using an online platform with no direct interaction from the interviewer and yet trying to elicit clinically relevant material in a parsimonious way. We acknowledge that (a) descriptions of processes common within persons may (sometimes) differ from those between persons; (b) several different disorders are being compared (and different pairs of disorders may be more or less similar); and (c) not only are several different disorders being compared, but also the clients described
TABLE 2 Categories of processes obtained after codification of participants' answers

\begin{tabular}{|c|c|}
\hline Categories & $\begin{array}{l}\text { No. of } \\
\text { sources }\end{array}$ \\
\hline Emotional & 35 \\
\hline Behavioural & 31 \\
\hline Self-self & 29 \\
\hline Self-other & 28 \\
\hline Insight and awareness & 28 \\
\hline Cognitive & 26 \\
\hline Skills and learning & 22 \\
\hline Establishing and maintaining therapeutic alliance & 22 \\
\hline Therapy/structure & 20 \\
\hline Body & 15 \\
\hline Trauma & 14 \\
\hline Family, couple and parenting & 11 \\
\hline Motivational & 10 \\
\hline Change consolidation & 8 \\
\hline
\end{tabular}

may have several diagnoses. Nonetheless, the bundle task served the purpose of eliciting a good dose of heterogeneous, pluralistic data.

To analyse the data, we used the qualitative analysis software QSR NVivo 11, which allows us to manage data and ideas, to formulate questions to the data, to create graphical models and to create a data report (Bazeley, 2007).

\section{3 | RESULTS}

Following the procedure described by Braun and Clarke (2006), we relied on participants' answers to extract information that allowed us to identify and characterise transdiagnostic processes. On average, participants used 304.92 words to answer our questionnaire $(S D=184.74$, Max $=735$, Min = 58). We further present the results of the thematic analysis.

\subsection{Common processes across disorders}

After analysing and codifying all data, a total of 14 categories of processes were created: emotional; behavioural; self-self; self-other; insight and awareness; cognitive; skills and learning; establishing and maintaining therapeutic alliance; therapy/structure; body; trauma; family, couple and parenting; motivational; and change consolidation.

Table 2 presents these categories and the total number of sources.

References and units of meaning coming from participants' answers allow for a more precise description of these categories. Each of them is comprised of some relevant subcategories of processes. Verbatim examples, coming from different participants, are 
presented for each category of processes, aiming to illustrate the subcategories involved.

Emotional processes are related to negative affect, emotional (dys)regulation, emotional expression, rejection and intolerance to emotional experiences, acceptance and attendance to emotional experiences, emotional knowledge, emotional contact or experiencing. For example, one participant mentioned: 'Common processes: poor emotion knowledge, heightened emotional arousal, high value for emotional stability, high expectations of self-regulation while they invalidate their emotional experiences, denial of distress despite lapses in socio-emotional functioning'. Another participant stated: 'Common process: extreme difficulty expressing emotions cathartically in appropriate contexts (i.e. laughing, crying at appropriate time)'.

Behavioural processes comprise behavioural tendencies such as addiction, aggression, compulsion, behavioural avoidance, behaviours caused by emotional arousal (e.g. self-harm), behavioural experiments and exposure, reinforcement, behavioural analysis, chain analysis and behavioural activation. For instance, one participant reflected: 'Concurrently I am addressing his distorted ideas about negative emotions, systematic avoidance of potentially anxiety provoking events, unrealistic expectations of emotional stability, escapist behaviours, poor emotion knowledge, and behavioural activation'. Another participant offered: 'The common process appears to be intolerance of discomfort of any kind, and a compulsive need to fix/check on (...)'.

Self-self processes include relational processes between parts of the self, self-perception, self-treatment, self-acceptance, sense of self and volition. One participant, for example, referred: 'I see in both women the same problem (...): a history of development that hampered the normal growth of self-confidence'. Another participant highlighted: 'The underlying theme is perfectionism. Thus self-criticism, fear of failure, needs to be observably competent'.

Self-other processes are linked to interpersonal difficulties and competences, communication style and skills, personal space and boundaries or lack thereof, safety, regulation of giving and receiving, regulation of autonomy and proximity, conflict/rupture and repair processes. For example, one participant mentioned: 'The underlying process for each appears to be interpersonal and affect avoidance. The restriction of interpersonal contact and avoidance of felt or expressed emotion in each promote the symptoms they experience'. Another participant stated: 'I am working with both of them on trying to recognise when they are engaging in mind-reading, to produce positive social behaviours, to learn better communication skills, and to de-catastrophise the possibility that others might find them worthy of criticism in some way'.

Insight and awareness processes are described as reflection, engaging in proximal or distal meaning-making, expanding awareness, exploring ambivalence, gaining insight and new perspective, self-monitoring, psychoeducation and mindfulness. For example, one participant expressed: 'Currently I am focused with both patients on the notion that thoughts are not facts, they do not tell the future and they are not accurate reflections of reality. They are not messages that must be attended to and just because they intrude or repeat, that does not mean they are important or valuable to explore'. Another participant shared: 'Helping the patient understand that the addictive behaviours are an ineffective way to cope with the symptoms of PTSD. Helping the patient replace the alcohol addiction behaviours with mindfulness, breathing to reduce stress/ anxiety of PTSD'.

Cognitive processes relate to thought processes, schemas and core beliefs, attentional processes, memory processes or reasoning processes. For example, one participant revealed: 'I think the underlying process in this client is thought rumination or preoccupation. $\mathrm{He}$ is constantly thinking about his girlfriend's behaviour and how she might cheat on him. This jealousy has persisted throughout all his romantic and sometimes even friendly relationships. He also ruminates a lot about things he might have done or said wrong in the past'. Another participant disclosed: 'The underlying process I think underlies both problems is her cognitive rigidity. It's something like if it was impossible to her to think more, deeper and differently in her own experience'.

Skills and learning processes were described in the scope of skill training and development, promoting a healthy lifestyle or practising new habits, a problem-solving orientation, or promoting adaptive ways of interpreting and responding. For example, one participant reflected that: 'In regards to both issues our work is heightened on enhancing her capacity to identify her internal resistance and to practice letting it go so that she might consider alternative responses'. Another participant identified: 'Teaching healthy lifestyle, implementing behavioural changes in lifestyle: sleep, nutrition, and exercise/movement. Education about how the brain functions and can be changed through self-directed positive neuroplasticity. Teach mindfulness and MBSR to develop self-regulation. Examine interpersonal relationships, increase awareness of negative impact of disorder on those relationships, and teach/practice communication and empathy to improve the quality of social connections'.

In what pertains to establishing and maintaining therapeutic alliance, data included processes such as enhancing motivation and collaboration, strains in the alliance, and identifying, addressing and exploring those ruptures. For example, one participant shared: 'In therapy, my fundamental concern in both cases is to attend to each patient's need for psychological safety within the therapeutic relationship'. Another participant stressed: 'In this phase the relationship (and analytically speaking the countertransference) is concordant: she is coming to therapy, helpful to have assistance, feeling empowered after the sessions and starting to get more everyday structure'.

Therapy/structure processes emerged in the context of therapeutic responsivity to the patient's variables or needs, as well as socialising the patient into the treatment process and format, together with its roles, rules and setting. For example, one participant expressed: 'Her self-rating and perfectionism hindered her previous therapy, because her doctoral student therapist used a reflective, non-directive approach to therapy which tended to upset her more and more as she described her symptoms. She sought a transfer, believing that she needed a more directive intervention style'. Another participant 
emphasised: 'They ask me for an intervention on the obsessive side of their pathology, rumination, worry, but have difficulties in working in a process way and continuously return to the interpretative way. We confronted each other and tried to find a common road together towards the symptom goals they asked me to help them solve'.

Body processes relate, for example, to somatic complaints or biological health issues, sleeping, nutrition, tension and relaxation, burnout, exercise and movement, and disconnection from body and sensations. For example, one participant stated: 'Common process: high somatic expression under emotional stress (i.e. dysregulation, involuntary movement of head, arms, legs)'. Another participant highlighted: 'I begin by educating my clients about the psychological and physical symptoms of trauma. Then I try to normalise exercise, sleep, and other physiological processes'.

Trauma processes were distinguished as being related to recent or early traumatic contexts, abuse of several types, extreme stress, loss, discrimination processes or harm reduction. For example, one participant expressed: 'All of my clients experience comorbid disorders stemming from Historic Trauma that is generational'. Another participant argued: 'Underlying common process, in my opinion, is extensive unprocessed trauma and loss, which by the way also underlies most or all of the symptoms that are common across diagnoses more generally'.

Family, couple and parenting processes included aspects such as parenting styles, abandonment by a caregiver or marital separation. For example, one participant shared: 'The common underlying issue is a dysfunctional family system that impaired interpersonal functionality in the two patients'. Another participant stressed: 'The common process in both disorders for this client is massive loss as a child, rejection by caregivers, isolation, withdrawal and inability to establish adult loving relationships'.

Motivational processes were comprised of aspects such as dissociation, devaluation, ineffective defences or experiential avoidance/interruption, ambivalence or resistance, and conflicting psychological needs. For example, one participant mentioned: 'The common process is experiential avoidance, and is hindering the clients, causing suffering because it keeps them focused on the problems, preventing decentration and liberation'. Another participant stated: 'A common underlying process is resistance to experience'.

Finally, change consolidation refers to processes such as empowerment, maintaining balance, processing the experience of change, repetition and ongoing exposure to new possibilities, and relapse prevention. For example, one participant shared: 'Another way working has been fostered has been enrolment in the medical clinic's rehabilitation program whereby psychological and physical difficulties are addressed holistically'. Another participant concluded: 'They feel better when they do such activities, but it is difficult to get them to maintain'.

\section{4 | DISCUSSION}

The great number of existing transdiagnostic models reflects the growing and incomplete state of this field. There are theoretical and empirical reasons to believe that there are important similarities between some disorders, with interventions that address these common processes potentially representing effective means (McEvoy, Nathan, \& Norton, 2009). The transdiagnostic approach-convergent, integrative and parsimonious-should be seen as an alternative approach, potentially complementary, that does not aim at replacing already existent specific approaches to each disorder-divergent, eclectic and creative (Clark, 2009; Mansell et al., 2009). Our exploratory study aimed at analysing which transdiagnostic processes emerged from the experiences of a sample of clinicians.

In terms of superordinate categories, the transdiagnostic processes originating from our sample's clinical focus and practice are the following: emotional; behavioural; self-self; self-other; insight and awareness; cognitive; skills and learning; therapeutic alliance; therapy/structure; body; trauma; family, couple, and parenting; motivational; and change consolidation.

In line with the idea that some processes are common to several disorders, some of the processes that were mentioned by previous authors were also present in our data. Emotional processes, for example, are described in several studies as being common to various clinical patterns (Barlow et al., 2011; McKay et al., 2011). Behavioural processes were also previously mentioned in the literature as having a transdiagnostic character (Harvey et al., 2004; McEvoy et al., 2009). Not surprisingly, cognitive processes also emerged as a category in our data, given their strong presence in the literature (Frank \& Davidson, 2014; Gellatly \& Beck, 2016; Harvey et al., 2004; McEvoy et al., 2009; McKay et al., 2011).

Other categories, though, are less frequently referred to as transdiagnostic in the literature, so attention should be paid to them in future studies, in order to clarify their nature (e.g. motivational processes or change consolidation processes). Moreover, when one considers the information in the subcategories of the transdiagnostic processes, somewhat different proposals or slightly new formulations can emerge.

As argued in Harvey and collaborators (2004) or Frank and Davidson (2014), transdiagnostic processes can inform about predisposing factors, precipitating factors and factors involved in the maintenance of a disorder or problem, whether they are contextual, biological or cognitive. Thus, there are various processes that can contribute to the expression of a clinically significant disorder. In line with this notion, we observed that some of the processes that participants shared reflect the predisposition of an individual to the development of certain difficulties (e.g. early trauma, parenting styles). Also in line with these authors, processes resulting from our data also differ in their nature. For example, while thought processes, attentional processes and beliefs are mainly of cognitive nature, others such as somatic complaints can be associated with a more biological nature. The process list is quite extensive and seems to merit further inquiry.

In terms of limitations to this study, it should be noted that the sample comprised a relatively small group of therapists who were interested in thinking about their practice using this type of scope. This might reflect a more integrative group of therapists who were most 
likely to accept taking part in this study. Also, most of them reported a great level of clinical experience and were highly qualified, coming from international professional societies in the field of psychotherapy, which might reflect more solidified methods of addressing the clinical reality or a predisposition to more readily and confidently focus on specific processes rather than others. In particular, we acknowledge that in several responses by our participants, it is possible to infer their specific training models and/or theoretical approaches. Despite our goal of obtaining naturalistic information based on the practitioners' practice, the open-ended questions in the context of our methodology might not have allowed us to go beyond standard case conceptualisation perspectives, even though the quantity and heterogeneity of participants and data are already informative for such a preliminary qualitative exploration. We also recognise that we could have presented an even more refined subcategorisation of the processes. We chose to focus on the more overarching ones, aiming for a parsimonious contribution to the field.

Altogether, future studies may use a different methodology (i.e. a more in-depth, extensive interview process) that may be better able to further capture psychotherapists' perspectives, explicitly exploring their uncertainties, struggles, nuances and reflective thinking on common processes between and within patients, as well as to better refine the characterisation of transdiagnostic processes from a naturalistic stance.

One could even go further as to inviting therapists to specifically try to reflect about general processes that they would identify as being more frequently present in their clinical caseload, independently of patients' disorders. Another line of questioning could involve asking clinicians to separately reflect on common processes that are more related to case conceptualisation or more linked to intervention aspects. Finally, in line with authors such as Mansell and collaborators (2009), who discuss the scientific approach of the transdiagnostic perspective as a more integrative one, it would also be interesting to explore the existence of any associations between clinicians' theoretical orientations or training trajectories and the quantity and nature of common processes that they identify or with which they usually work.

We hope that this study contributes to the growth and evolution of transdiagnostic theory and practice, and that it works as an inspiration source for future studies that can contribute even more to the development not only of transdiagnostic approaches, but also of more naturalistic and pluralistic conceptualisations of psychopathology. We also hope that future research efforts can remain as inclusive as possible, listening to psychotherapists' voices while being attentive to factors related to their theoretical orientations or those of the researchers conducting the study.

\section{5 | CONCLUSION}

Transdiagnostic processes (common to different disorders within or between clients) may serve as a complementary guide to clinical conceptualisation and intervention. Our study aimed at linking theory to clinical reality by addressing clinicians on this topic and on their representations of these types of common processes. The authors' analysis of clinicians' representations revealed several categories, some of which had been commonly discussed in the literature on transdiagnostic treatments and processes, while others were relatively novel. Overall, this study aims to configure an additional clarification of psychopathological constructs, relying on natural language from practising clinicians to examine potential transdiagnostic processes. Not surprisingly, the resultant qualitative data, even though preliminary, point to the need of future studies on transdiagnostic processes to embrace a pluralistic, integrative route.

\section{ACKNOWLEDGMENTS}

There was no funding for this study. The authors would like to acknowledge all participants who voluntarily took part in the study.

\section{ORCID}

António Farinha-Fernandes (iD https://orcid. org/0000-0002-6136-8410

Nuno Conceição iD https://orcid.org/0000-0001-8038-5841

\section{REFERENCES}

Baer, R. A. (2007). Mindfulness, assessment, and transdiagnostic processes. Psychological Inquiry, 18(4), 238-242. https://doi. org/10.1080/10478400701598306.

Barlow, D. H., Ellard, K. K., Fairholme, C. P., Farchione, T. J., Boisseau, C. L., Allen, L. B., \& Ehrenreich-May, J. (2011). The unified protocol for transdiagnostic treatment of emotional disorders: Client workbook and therapist guide. New York: Oxford University Press.

Bazeley, P. (2007). Qualitative data analysis with NVivo. London: SAGE Publications

Braun, V., \& Clarke, V. (2006). Using thematic analysis in psychology. Qualitative Research in Psychology, 3(2), 77-101. https://doi. org/10.1191/1478088706qp063oa.

Carey, T. A. (2008). Perceptual control theory and the method of levels: Further contributions to a transdiagnostic perspective. International Journal of Cognitive Therapy, 1(3), 237-255. https://doi.org/10.1521/ ijct.2008.1.3.237.

Clark, D. A. (2009). Cognitive behavioral therapy for anxiety and depression: Possibilities and limitations of a transdiagnostic perspective. Cognitive Behaviour Therapy, 38, 29-34. https://doi. org/10.1080/16506070902980745.

Dalgleish, T., Black, M., Johnston, D., \& Bevan, A. (2020). Transdiagnostic approaches to mental health problems: Current status and future directions. Journal of Consulting and Clinical Psychology, 88(3), 179-195. https://doi.org/10.1037/ccp0000482.

Dudley, R., Kuyken, W., \& Padesky, C. A. (2011). Disorder specific and trans-diagnostic case conceptualization. Clinical Psychology Review, 31(2), 213-224. https://doi.org/10.1016/j.cpr.2010.07.005.

Ellis, A. (1962). Reason and emotion in psychotherapy. Oxford: Lyle Stuart.

Feldman, J. S., Tung, I., \& Lee, S. S. (2017). Social skills mediate the association of ADHD and depression in preadolescents. Journal of Psychopathology and Behavioral Assessment, 39(1), 79-91. https://doi. org/10.1007/s10862-016-9569-3.

Frank, R. I., \& Davidson, J. (2014). The transdiagnostic road map to case formulation and treatment planning: Practical guidance for clinical decision making. Oakland, CA: New Harbinger Publications.

Gallagher, M. W. (2017). Transdiagnostic mechanisms of change and cognitive-behavioral treatments for PTSD. Current Opinion in Psychology, 14, 90-95. https://doi.org/10.1016/j.copsyc.2016.12.002. 
Gellatly, R., \& Beck, A. T. (2016). Catastrophic thinking: A transdiagnostic process across psychiatric disorders. Cognitive Therapy and Research, 40(4), 441-452. https://doi.org/10.1007/s10608-016-9763-3.

Harvey, A. G., Watkins, E., Mansell, W., \& Shafran, R. (2004). Cognitive behavioural processes across psychological disorders: A transdiagnostic approach to research and treatment. Oxford, UK: Oxford University Press.

Helzer, J. E., Kraemer, H. C., \& Krueger, R. F. (2006). The feasibility and need for dimensional psychiatric diagnoses. Psychological Medicine, 36(12), 1671-1680. https://doi.org/10.1017/S0033 29170600821X.

Hengartner, M. P., \& Lehmann, S. (2017). Why psychiatric research must abandon traditional diagnostic classification and adopt a fully dimensional scope: Two solutions to a persistent problem. Frontiers in Psychiatry, 8, 101. https://doi.org/10.3389/fpsyt.2017.00101.

Hill, C., Chui, H., \& Baumann, E. (2013). Revisiting and reenvisioning the outcome problem in psychotherapy: An argument to include individualized and qualitative measurement. Psychotherapy, 50(1), 68-76. https://doi.org/10.1037/a0030571.

Hopwood, C. J., Kotov, R., Krueger, R. F., Watson, D., Widiger, T. A., Althoff, R. R., ... Zimmermann, J. (2018). The time has come for dimensional personality disorder diagnosis. Personality and Mental Health, 12(1), 82-86. https://doi.org/10.1002/pmh.1408.

Kraemer, H. C., Noda, A., \& O'Hara, R. (2004). Categorical versus dimensional approaches to diagnosis: Methodological challenges. Journal of Psychiatric Research, 38(1), 17-25. https://doi.org/10.1016/ S0022-3956(03)00097-9.

La Greca, A., Ehrenreich-May, J., Mufson, L., \& Chan, S. F. (2016). Preventing adolescent social anxiety and depression and reducing peer victimization: Intervention development and open trial. Child \& Youth Care Forum, 45(6), 905-926. https://doi.org/10.1007/ s10566-016-9363-0.

LaRowe, L. R., Zvolensky, M. J., \& Ditre, J. W. (2019). The role of anxiety-relevant transdiagnostic factors in comorbid chronic pain and tobacco cigarette smoking. Cognitive Therapy and Research, 43, 102113. https://doi.org/10.1007/s10608-018-9957-y.

Mansell, W., Harvey, A., Watkins, E., \& Shafran, R. (2009). Conceptual foundations of the transdiagnostic approach to CBT. Journal of Cognitive Psychotherapy, 23(1), 6-19. https://doi.org/10.1891/0889-8391.23.1.6.

McEvoy, P. M., Nathan, P., \& Norton, P. J. (2009). Efficacy of transdiagnostic treatments: A review of published outcome studies and future research directions. Journal of Cognitive Psychotherapy, 23(1), 20-33. https://doi.org/10.1891/0889-8391.23.1.20.

McKay, M., Fanning, P., \& Ona, P. Z. (2011). Mind and emotions: A universal treatment for emotional disorders. Oakland, CA: New Harbinger Publications.

Nelson, B., McGorry, P. D., Wichers, M., Wigman, J. T. W., \& Hartmann, J. A. (2017). Moving from static to dynamic models of the onset of mental disorder: A review. JAMA Psychiatry, 74(5), 528-534. https:// doi.org/10.1001/jamapsychiatry.2017.0001.

Nolen-Hoeksema, S., \& Watkins, E. R. (2011). A heuristic for developing transdiagnostic models of psychopathology: Explaining multifinality and divergent trajectories. Perspectives on Psychological Science, 6(6), 589-609. https://doi.org/10.1177/1745691611419672.

Rodriguez-Seijas, C., Eaton, N. R., \& Krueger, R. F. (2015). How transdiagnostic factors of personality and psychopathology can inform clinical assessment and intervention. Journal of Personality Assessment, 97(5), 425-435. https://doi.org/10.1080/00223891.2015.1055752.

Sauer-Zavala, S., Gutner, C. A., Farchione, T. J., Boettcher, H. T., Bullis, J. R., \& Barlow, D. H. (2017). Current definitions of 'transdiagnostic' in treatment development: A search for consensus. Behavior Therapy, 48(1), 128-138. https://doi.org/10.1016/j.beth.2016.09.004.
Taylor, S., \& Clark, D. A. (2009). Transdiagnostic cognitive-behavioral treatments for mood and anxiety disorders: Introduction to the special issue. Journal of Cognitive Psychotherapy, 23(1), 3-5. https://doi. org/10.1891/0889-8391.23.1.3.

Widiger, T. A. (1992). Categorical versus dimensional classification: Implications from and for research. Journal of Personality Disorders, 6(4), 287-300. https://doi.org/10.1521/pedi.1992.6.4.287.

Yee, C. M., Javitt, D. C., \& Miller, G. A. (2015). Replacing DSM categorical analyses with dimensional analyses in psychiatry research: The Research Domain Criteria Initiative. JAMA Psychiatry, 72(12), 11591160. https://doi.org/10.1001/jamapsychiatry.2015.1900.

Zelkowitz, R. L., \& Cole, D. A. (2019). Self-criticism as a transdiagnostic process in nonsuicidal self-injury and disordered eating: Systematic review and meta-analysis. Suicide and Life-Threatening Behavior, 49(1), 310-327. https://doi.org/10.1111/sltb.12436.

\section{AUTHOR BIOGRAPHIES}

António Farinha-Fernandes is a Clinical and Health Psychologist (MSc) with training in cognitive-behavioural and integrative counselling/psychotherapy. He develops his professional activity in clinical work with adults and adolescents, both at Faculdade de Psicologia, Universidade de Lisboa and in a private practice setting. In parallel, he collaborates in research projects in the fields of Cognitive Psychology and Counselling/Psychotherapy, in an effort to contribute to the development of both clinical practice and theory.

Nuno Conceição is a broadly trained clinician, working from an integrative psychotherapy perspective. He has been practising psychotherapy with adults since 1999 and half of his professional life is dedicated to his clients. He also consults and teaches as Guest Assistant Clinical Professor at Faculdade de Psicologia, Universidade de Lisboa since 2011. He directs the Paradigmatic Complementarity Lab, where he conducts research on Psychotherapy Integration and trains clinicians at the Faculty's Community Service. He is also a professional therapist trainer on Psychotherapy Integration modules at professional societies. He was pastpresident of the Society for the Exploration of Psychotherapy Integration.

Rita Silva is currently working in the Human Resources Management area, with a special focus on emotional intelligence, transformational leadership, work life balance, employee engagement and work culture. She is also interested in the fields of effective communication, ethical practice, cross-cultural awareness and relationships management. She completed her Master's Degree in Clinical and Health Psychology (CognitiveBehavioural and Integrative Psychotherapy) in 2016 at Faculdade de Psicologia, Universidade de Lisboa. Her dissertation was focused on the transdiagnostic approach. 